\title{
Challenges Faced During Primary Total Knee Arthroplasty in Rheumatoid Knee in Females in the 5th Decade with Severe Osteoporosis
}

\begin{abstract}
Introduction: Rheumatoid Arthritis [RA] is a chronic inflammatory disorder of the synovium resulting in joint destruction. The knee is among the most commonly affected joints in RA and advanced arthritis of the involved joint is associated with poor prognosis contributing to patient pain and disability. In advanced disease, Total Knee Arthroplasty [TKA] has proven to be the most successful intervention that reduces knee pain and improves physical function in RA patients.

Aim: The aim of our study was to correlate the pre-operative radiographic findings of advanced knee rheumatoid arthritis with the intraoperative challenges and critical evaluation of intraoperative findings in lieu of severe osteoporosis.

Materials and methods: A total of 30 total knee arthroplasties were performed for 24 females belonging to the fifth decade in a tertiary care centre for rheumatoid arthritis of the knee joint with a posterior stabilized knee. Patients were regularly followed up and were assessed at the end of 5 years for the function of the knee joint, quality of life, ability to walk and perform routine daily activities, local signs of infection, activity of the disease and general condition.

Results and conclusion: Thorough pre-operative evaluation along with awareness about the possible intra-operative complications and challenges should be borne in mind while performing total knee arthroplasty in rheumatoid knees. No article till date has mentioned about the intra-operative challenges faced during total knee arthroplasty in rheumatoid knee.
\end{abstract}

Keywords: Total knee arthroplasty; Rheumatoid arthritis; Knee

\section{Introduction}

Rheumatoid Arthritis [RA] is a chronic inflammatory disorder of the synovium resulting in joint destruction. The knee is amongst the most commonly affected joints in RA and it is estimated that up to $90 \%$ of patients with RA will eventually have the involvement of the knees [1]. Over the past three decades there has been a considerable improvement with respect to advances in understanding the pathogenesis and treatment of RA and with the advent of highly effective biologic therapies, fewer individuals with RA suffer endstage joint destruction [2].

Despite this success, approximately $20-25 \%$ of afflicted individuals develop advanced arthritis of the affected joints contributing to patient pain and overall disability [3]. The prognosis is poor with $80 \%$ of patients being disabled 20 years from primary diagnosis resulting in gross compromise of occupational activities as well as activities of daily living [4].

In advanced disease, Total Knee Arthroplasty [TKA] has proven to be the most successful intervention that reduces knee pain and improves physical function in RA patients. RA patients carry additional potential for peri and post-operative complications; hence considerations regarding preoperative evaluation and surgical

\section{Journal of Orthopedics \& Rheumatology}

\section{Sharath KR* and Rahul Hemant Shah}

Department of Orthopedics, Ramaiah Medical College \& Hospitals, India

*Address for Correspondence

Dr. Sharath KR, Department of Orthopedics, Ramaiah Medical College \& Hospitals, Bengaluru, India E-mail: dr.sharathkr@gmail.com

Submission: 05 August, 2018

Accepted: 07 September, 2018

Published: 17 September, 2018

Copyright: ๑ 2018 Ramanath SK, et al. This is an open access article distributed under the Creative Commons Attribution License, which permits unrestricted use, distribution, and reproduction in any medium, provided the original work is properly cited.

techniques must be taken into account in order to improve the results of TKA [5]. This is the first article of its kind where we shall discuss about the intra-operative challenges we faced while performing total knee arthroplasty in females of the 5th decade.

\section{Materials and Methods}

Between March 2012 to July 2014 a total of 30 TKA were performed on 24 females in a tertiary care centre for rheumatoid arthritis of the knee joint. The age of the patients ranged between 41 years to 48 years with a mean age of 44.29 years. Out of the 30 operated knees, 19 were left knee and 11 were right knee.

6 patients underwent bilateral TKAs, both in different sittings. There was a gap of 2 months between the first and the second knee in all the 6 cases of bilateral TKA. All the patients were diagnosed with Rheumatoid arthritis prior to planning for the procedure and the duration between diagnosis and procedure ranged from 3 years to 11 years with an average of 5.95 years. Out of the 24 patients, 12 patients had radiological evidence of arthritis of either of the hip joints on presentation. Apart from the fact that all of the presented patients were on non-steroidal anti-inflammatory drugs [NSAIDs], 20 patients were on Disease Modifying Anti-Rheumatic Drugs [DMARDs] prior to surgery and 4 patients had never been treated for Rheumatoid arthritis before. No patients were on biological response modifiers. 12 out of 20 patients on DMARDs were also on Glucocorticoid Therapy. The DMARDs [Methotrexate/Sulfasalazine/ Hydroxychloroquine] were withheld at least 48 hours prior to surgery and started 3 days after surgery in all patients. Perioperative dose adjustment of glucocorticoids was done for needed patients to prevent Addisonian crisis. Diabetes, hypertension and hypothyroidism were the common associated medical co-morbidities of the patients. All the patients were thoroughly evaluated clinically and radiologically and planned for total knee arthroplasty following necessary medical clearance.

All patients were given one pre-operative dose of antibiotic [Inj. Cefuroxime $1.5 \mathrm{gms}$ ] and the same was given post operatively for two 
Citation: Sharath KR, Shah RH. Challenges Faced During Primary Total Knee Arthroplasty in Rheumatoid Knee in Females in the 5th Decade with Severe Osteoporosis. J Orthopedics Rheumatol. 2018; 5(2): 4

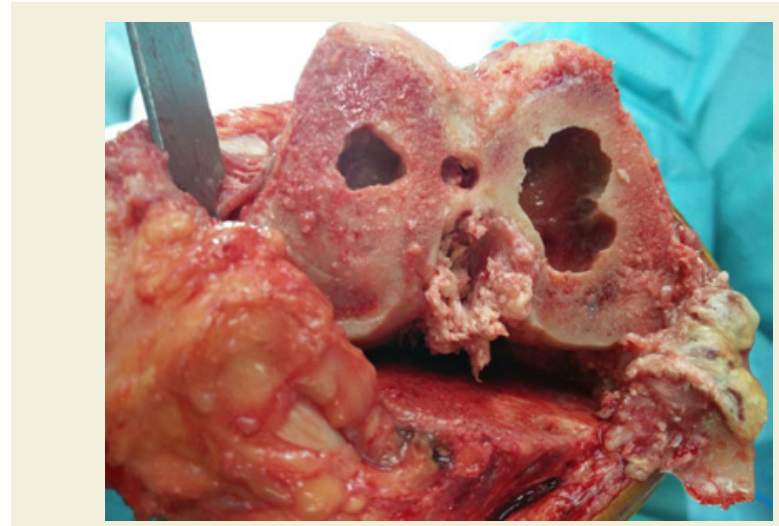

Figure 1: Intra operative picture showing cyst in femoral condyle.

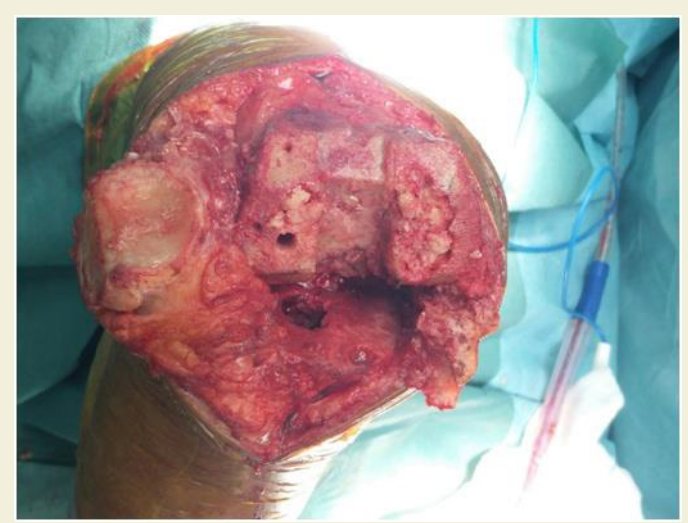

Figure 2: Cyst has been filled with impaction bone grafting.

days. All patients were operated by the same team of surgeons using standard medial para patellar approach with posterior stabilized knee. Post operatively surgical site drain was removed on the first postoperative day and the patients were made to walk full weight bearing with the help of walker. Dressings were changed at regular intervals and observed for any soakage or discharge. Anti-DVT measures were also taken.

Patients were regularly followed up and were assessed at the end of 5 years for the function of the knee joint, quality of life, ability to walk and perform routine daily activities, local signs of infection, activity of the disease and general condition.

\section{Results}

The results are mentioned tabulated below. The difference in the average knee society score and the difference in the average knee available range of motion were found to be statistically significant (Table 1).

\section{Data analysis}

Descriptive statistics was analyzed with SPSS version 20.0 software. Descriptive statistics of Knee society score and available range of knee motion were analysed and presented in terms of mean with standard deviation. Paired-T test was used to compare the scores before and after the procedure. $\mathrm{P}$ value $<0.05$ was considered statistically significant.

Thickness of patella was less than $20 \mathrm{~mm}$ in 20 patients. Overgrowth of synovial fold over the patellar cartilage was noticed in 7 patients. This warranted complete excision of the synovium for better post-operative outcomes.

17 patients were noticed to have stiff knee pre-operatively. Preoperative radiographs showed obliteration of patellofemoral joint space which was confirmed after medial parapatellar approach. We noticed fusion of the patella over the femoral condyle. Movement could be achieved upon osteotomized patella over the femoral condyle.

Appearance of cysts following bone cuts were also one of the common intra-operative findings which was noted. Cysts were mostly found in femoral condyles which required impaction bone grafting (Figures 1 and 2). Resected bone was used as bone graft. In our study we encountered cysts in ten knees. Defects in proximal tibia were not common. One patient had severe postero-medial tibial condylar defect which needed tibial stem and wedge application.

\section{Discussion}

RA patients are often younger by approximately 10 or more years than osteoarthritis OA patients at the time of TKA. Following the advent of recent medication for RA, the life expectancy for RA patients can be compared to the general population and hence the best possible implantation technique should be used. Bone quality is generally poor in RA patients. This poor bone quality occurs due to the combined effect of inflammatory disease process itself, disuse of the part of the body due to pain and chronic use of steroids. For these reasons, in RA patients, cemented TKA is preferred over uncemented or hybrid TKA [6-8].

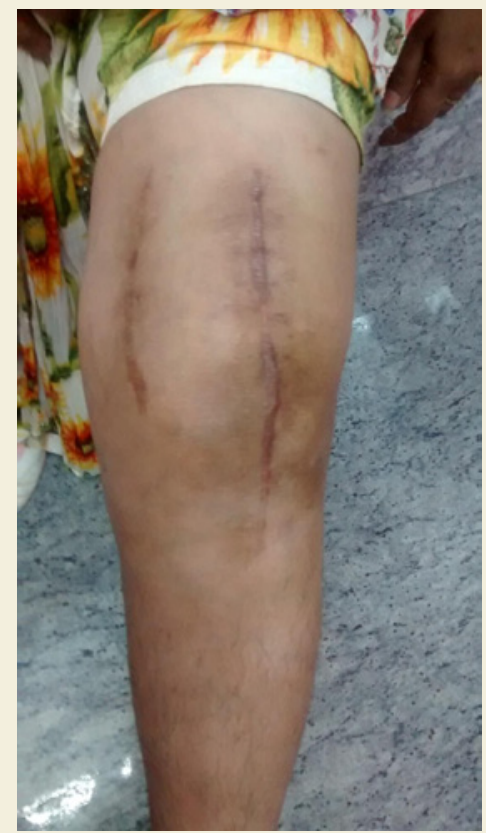

Figure 3: Previous paramedian scar showing open synovectomy and midline incision of knee replacement. 
Table 1: Pre and post-operative knee society score and $P$ values.

\begin{tabular}{|l|c|c|}
\hline & Pre -operative & Post-Operative (Follow up at 5 years) \\
\hline Average Knee Society Score & 55.64 & 76.79 \\
\hline Average Knee ROM & 50.88 & \multicolumn{1}{|c|}{ palue } \\
\hline No. of patients with knee flexion $<\mathbf{9 0 ~ d e g}$ & 22 & $<0.05$ \\
\hline
\end{tabular}

RA knees are notorious with respect to flexion deformity when compared to OA knees. It is necessary to treat these deformities by posterior soft tissue release rather than simple removal of additional distal femoral bone as increased distal femoral resection would elevate the joint line. In contrast to OA patients in whom an almost complete resolution of flexion contracture is required at the time of surgery, RA patients undergoing TKA can be left accepted with mild post-operative flexion deformity $[9,10]$.

One patient who was operated for total knee arthroplasty had severe ipsilateral ankle arthritis with planovalgus foot deformity. Rehabilitating the patient was difficult due to the ankle pathology and pantalar arthritis. We recommend suggestions of Wilkinson et al. for surgical sequence for lower limb reconstruction: forefoot, hip, knee, hind foot and then ankle, which they deemed the order of "reliability" of the procedures and ease of rehabilitation [11].

Various authors have expressed their concerns regarding substituting or retaining Posterior Cruciate Ligament (PCL) in patients with RA. Though PCL is extra synovial and RA is a synovial pathology, long time destruction of joint and pannus formation might lead to destruction of PCL leading to knee instability. Laskin reviewed 178 RA patients at an average of 8.2 years follow-up and demonstrated a $50 \%$ instability rate with PCL retaining implants in contrast to a $1 \%$ instability rate with the PCL sacrificing implants [12]. Goldberg et al. and Kristensen et al. demonstrated a $0 \%$ to $14 \%$ instability rate for PCL sacrificing implants respectively [7,12]. Gill et al. and Meding et al have also shown similar rates of instability for PCL retaining implants (1.5\% and 9.9\% respectively) [13].

We faced various challenges intra-operatively. Meng $\mathrm{XH}$ et al conducted a prospective study of 26 knee joints in 22 patients [14] The stage of knee joints in RA patients was assessed based on the evaluation criteria of Larsen et al. In their study, the extent of articular destruction was classified as grade 4 for the medial and lateral femoral condyles and the medial and lateral tibial plateaus for most patients, with an average destruction grade of $3.6(\mathrm{~F}=5.455, \mathrm{P}=0.002)$, with the least amount of destruction identified on the lateral femoral condyle. The majority of knee joints in the RA patients were at stage $3(21 / 26$, $80.8 \%)$, followed by stage $4(4 / 26,15.4 \%)$ [14]. Punctate hemorrhages over femoral condyle and patchy destruction of the condyles was noted in 21 of the 24 patients in our study. This correlated with gross osteoporosis noted on pre-operative radiographs of the knee. These patients were operated taking conservative cuts over both, the femoral as well as the tibial side. No bone grafts or wedges were needed for these cases.

$30-50 \mathrm{ml}$ of outpouring of synovial fluid was noted in 20 patients upon performing an anteromedial arthrotomy. The synovial fluid was sent for analysis for all the 20 patients. The cell counts ranged from 2,000 to 11,000 with an average value of $6,500 / \mathrm{mm}^{3}$. All the samples were negative on Gram's as well as Ziehl-Neelsen stain and had no growth on culture media.
Eversion of patella is another challenge while performing total knee arthroplasty in rheumatoid knee. This step has to be done with utmost care as the soft tissue is of friable quality. 1 patient had partial peeling $(4 \mathrm{~mm})$ of the patella tendon from the tibial side. We repaired the peeled part immediately after implantation and the patient was put on a long knee brace for a period of 6 weeks. Patient was started on toe-touch weight bearing with the help of a height adjustable walker from the third week after the surgery. Knee range of motion was initiated after 6 weeks although static quadriceps and hamstring exercises were started from the first day after surgery. In order to avoid peeling of the patella tendon, always subluxate the patella with a straight Hohmann's retractor.

Visualization of posterolateral tibia was difficult in 10 patients. Synovectomy around the meniscus and posterolateral part of the tibia helped in better visualization of the tibial condyles as this facilitated anterior subluxation of the tibia which helped in tibial component and poly placement.

We noted 3 cases of peeling of collateral ligaments with the epicondyle attached from the femoral side (1 medial and 2 lateral collateral ligaments). The peeled off bony attachments were fixed to the femur using $2.4 \mathrm{~mm}$ screws in all the 3 cases. These patients were put on strict non-weight bearing regime for the first 3 weeks which was eventually made partial weight were bearing and then full-weight bearing.

Jujo $\mathrm{Y}$ et al. retrospectively analyzed 329 TKAs performed in 230 female patients with rheumatoid arthritis [15]. The mean age was 61.8 years, and the mean follow-up period was 6.2 years. Patellar resurfacing was performed in all cases. Five postoperative patellar fractures $(1.51 \%)$ were identified, and a thin residual patellar thickness and the use of Posterior-Stabilizing (PS) components were identified as significant risk factors, although the number of fractures was small in both groups. Patellar thickness was significantly lower in the group with than without fracture (Mean, 10.4 vs. $12.2 \mathrm{~mm} ; \mathrm{P}=0.01$ ), and the proportion with PS was significantly higher in the group with fracture ( $\mathrm{P} \mathrm{b}=0.001)$ [15]. We noticed that the thickness of patella was less than $20 \mathrm{~mm}$ in 20 patients. However, no patient had patella thickness less than $15 \mathrm{~mm}$. No intra-operative or post-operative patellar fractures were noted in our study. Overgrowth of synovial fold over the patellar cartilage was noticed in 7 patients. This warranted complete excision of the synovium for better post-operative outcomes.

17 of our patients were noticed to have stiff knee pre-operatively. Pre-operative radiographs showed obliteration of patellofemoral joint space which was confirmed after medial parapatellar approach. We noticed fusion of the patella over the femoral condyle. Movement could be achieved upon osteotomizing patella over the femoral condyle.

Appearance of cysts following bone cuts were also one of the common intra-operative findings which was noted. Cysts were mostly 
Citation: Sharath KR, Shah RH. Challenges Faced During Primary Total Knee Arthroplasty in Rheumatoid Knee in Females in the 5th Decade with Severe Osteoporosis. J Orthopedics Rheumatol. 2018; 5(2): 4

found in femoral condyles which required impaction bone grafting (Figures 1 and 2). Resected bone was used as bone graft. In our study we encountered cysts in ten knees. Defects in proximal tibia were not common. One patient had severe posteromedial tibial condylar defect which needed tibial stem and wedge application.

In RA patients, management of synovium during TKA has also been a concern. In the presence of active inflammatory synovitis, complete synovectomy is recommended because recurrent synovitis after TKA may occur in this patient group and complete synovectomy in such patients may lead to improvement knee pain [16]. However, limited synovectomy should be considered in the presence of inactive, quiet synovitis as it may induce fibrotic reaction. Three patients underwent open synovectomy before TKA. Previously, medial parapatellar skin incision was used. We used midline incision for TKA. Gap between two were $5 \mathrm{~cm}$ (Figure 3). There were no wound complications.

In fixed valgus knees, the lateral structures, including lateral collateral ligaments, iliotibial band, joint capsule and popliteus are contracted with relative laxity of the medial structures. Fixed valgus is common in rheumatoid knees. Adequate gradual lateral release is critical. Correction of such deformity usually consists of gradual release, such as in the pie-crust technique, progressing from division of the iliotibial band to the lateral collateral ligament at its femoral origin.

One patient in our study developed discharging sinus from the operated knee following redness and swelling after 9 months of surgery. The patient was on glucocorticoid therapy before and after the procedure. The pus sample from the knee was sent for microbiological investigation and methicillin sensitive Staphylococcus aureus was grown. Arthrotomy and debridement was done and prolonged appropriate antibiotic was administered for 6 weeks. Since patient did not give consent for two staged revision surgery, regular dressing for sinus and wound care was advised.

Two patients had brown coloured thick synovial fluid collection inside the joint intraoperatively. Intra-operative grams staining showed gram positive cocci in clusters and hence arthroplasty was abandoned. Complete synovectomy was done and supported with appropriate post-operative antibiotics for 3 weeks. TKA was done for one of them and another was lost to follow up.

\section{Strengths}

There are multiple articles related to rheumatoid arthritis in knee which compare about the long term outcomes of cemented versus uncemented TKA. Many articles also compare about the outcomes of PCL retaining versus PCL substituting. The post-operative complications of TKA have been well documented in multiple articles. However, no article to date has mentioned about the intra-operative challenges faced while performing TKA in rheumatoid knees. This is the first article of its kind which not only mentions about these challenges but also mentions about the necessary measures to be taken in order to avoid them and the treatment of these complications in the unfortunate event if these complications were to happen.

\section{Weaknesses}

The major weakness of this study is the small sample size. One more weakness of our study is the short duration of follow-up period which is 5 years. A longer follow-up period will give a better idea about the clinical status of the patient after the surgery.

\section{Conclusion}

Knee replacement for arthritis of knee secondary to rheumatoid disease is a time tested procedure and results are promising. There is a significant improvement in functional scores of the knee after TKA. Thorough pre-operative evaluation is mandatory for successful outcomes after the surgery. However, one should be aware about the possible intra-operative challenges and complications that may occur in these patients and appropriate management is needed for the same to have good post-operative outcomes.

\section{References}

1. Kajino A, Yoshino S, Kameyama S, Kohda M, Nagashima S (1997) Comparison of the results of bilateral total knee arthroplasty with and without patellar replacement for rheumatoid arthritis. A follow-up note. J Bone Joint Surg Am 79: 570-574

2. da Silva E, Doran MF, Crowson CS, O'Fallon WM, Matteson EL (2003) Declining use of Orthopedic Surgery in patients with rheumatoid arthritis? Results of a long-term population based assessment. Arthritis Rheum 49: 216-220.

3. Tanaka E, Saito A, Kamitsuji S, Yamada T, Nakajima A, et al. (2005) Impact of shoulder, elbow and knee joint involvement on assessment of rheumatoid arthritis using the American College of Rheumatology Core Data Set. Arthritis Rheum 53: 864-871.

4. Laskin RS, O'Flynn HM (1997) The Insall Award. Total knee replacement with posterior cruciate ligament retention in rheumatoid arthritis. Problems and complications. Clin Orthop Relat Res 345: 24-28.

5. Momohara S, Inoue E, Ikari K, Kawamura K, Tsukahara S, et al. (2007) Risk factors for total knee arthroplasty in rheumatoid arthritis. Mod Rheumatol 17: 476-480.

6. Chmell MJ, Scott RD (1999) Total knee arthroplasty in patients with rheumatoid arthritis. An overview. Clin Orthop Relat Res 366: 54-60.

7. Kristensen O, Nafei A, Kjaersgaard-Andersen P, Hvid I, Jensen J (1992) Long-term results of total condylar knee arthroplasty in rheumatoid arthritis. J Bone Joint Surg $\mathrm{Br} 74:$ 803-806.

8. Ranawat CS, Padgett DE, Ohashi Y (1989) Total knee arthroplasty for patients younger than 55 years. Clin Orthop Relat Res 248: 27-33.

9. Scott RD, Sarokhan AJ, Dalziel R (1984) Total hip and total knee arthroplasty in juvenile rheumatoid arthritis. Clin Orthop Relat Res 182: 90-98.

10. Chung HK, Choi YJ, Choi CH, Kim JH, Roh KW (1999) Radiologic evaluation of improved residual flexion contracture after TKR in rheumatoid arthritis. Knee Surg Relat Res 11: 26-31.

11. Wilkinson JM, Stanley D, Getty CJ (2004) Surgical management of the rheumatoid patient. Curr Orthop 18: 357-370.

12. Goldberg VM, Figgie MP, Figgie HE $3^{\text {rd }}$, Heiple KG, Sobel M (1988) Use of a total condylar knee prosthesis for treatment of osteoarthritis and rheumatoid arthritis. Long-term results. J Bone Joint Surg Am 70: 802-811.

13. Gill GS, Joshi AB (2001) Long-term results of retention of the posterior cruciate ligament in total knee replacement in rheumatoid arthritis. J Bone Joint Surg Br 83: 510-512.

14. Meng XH, Wang Z, Zhang XN, Xu J, Hu YC (2018) Rheumatoid arthritis of knee joints: MRI-pathological correlation. Orthop Surg 10: 247-254.

15. Jujo Y, Yasui T, Nagase Y, Kadono Y, Oka H, et al. (2013) Patellar fracture after total knee arthroplasty for rheumatoid arthritis. J arthroplast 28: 40-43.

16. Jensen CM, Poulsen S, Ostergren M, Hansen KH (1991) Early and late synovectomy of the knee in rheumatoid arthritis. Scand J Rheumatol 20: 127131. 